180

ANT LBUUY RESPONSE IN NEUBORNS TO DIFFERENT DOSAGES OF HEPATITIS B VACCINES.

C Rosendahl, $F$ Deinhardt (introduced by KP Riegel). Kindefpoliklinik and Max v. Pettenkofer Institut, University of Munich, FRG.

Since the avalability of the active hepatitis $B$ vaccine in addition to hepatitis $B$ immunoglobulin it became possible to effectively interrt spt the potential transmission of virus from a pregnant carrier mother to her newborn. All babies $(n=42)$ of HBsAg carrier mothers delivering at the Munich University's women hospital and several city hospitals in Bavaria between october 1982 and december 1983 were given passive immunisation with $1 \mathrm{ml}$ hepatitis $B$ immunoglobulin and simultaneous active vaccination with $1 \mathrm{ml}$ of the hepatitis $B$ vaccine (containing $20 \mathrm{Hg}$ HBSAg in $1 \mathrm{ml} ; \mathrm{H}-\theta-\operatorname{Vax}(R))$. Since january 1984 half the previoust ly recommended dosis ( $10 \mu \mathrm{HBsAg}$ ) has been administered to a further 35 infants. Finally with the beginning of this year some newborns at these hospitals will receive $5 \mu \mathrm{HBsAg}$ of the recombinant yeast-vaccine (MSD laboratories). Infants' serum samples were collected at birth, at the 2 nd and 6 th months of age (when the publicly recommended routing sheck-ups take place), and at the end of the first and second year of life. Results: All vaccinated newborns developed protecting antibody levels at a time when passively transferred anti-HBs has usually disappeared. The mean antibody-titer in the $20 \mu \mathrm{g}$ group at the time of the third booster was higher than in the $10 \mu \mathrm{g} g r o u p$, but not significant. ly so. The new recombinant vaccine has been administered to 2 newborns as yet and their antibody response remains to be seen. Since the 2.5 years of the study anti-HBs fell below the protective level of $10 \mathrm{mIU} /$ $\mathrm{ml}$ in two children only. These data suggest that a vaccine dosis of $10 \mu \mathrm{H}$ HsAg might be as effective as the previously used $20 \mu \mathrm{g}$ dosis in most children.

\section{VACCINE ASSOCIATED POLIOMYELITIS}

181 Mifectious Diseases and Pediatil Matcitcher $(3)$. Mepartments $(1,2)$ Shaare Zedek Medical Center, Jerusalem, and Central Virology Laboratory, TelHashomer (3), Israel.

A 3 month old girl who developed paralytic poliomyelitis three weeks after the first oral dose of Sabin polio vaccine is described. After a three days episode of viral meningitis the child developed subsequently a quadriplegic form of poliomyelitis with facial nerve involyement. All three types of vaccine polio virus were isolated from the stool for a two month period. The child developed high antibody titers to all three types of poliovirus. The pathophysiology epidemology laboratory problems as well as preventive measures in case of vaccine associated poliomyelitis are presented. 183 Septicemia in the Newtorn Intensive Care Lhit (NIOU) caused by Vogel M, Roos R, Fahmow IM, Sengespeick C, Kraeft H

v.Haunersches Kinderspital of the Munich University,Lindwurmstr 4, -8000 Minchen 2 During 1984 nine of the 135 patients (pts) admitted to our NId had 11 episodes of septit cemia with Staph epi. In the last 5 years we had $5.2 \pm 3.6$ septicenias per year in pts with a central venous catheter and of those the majority i.e. $3.4+3$ were caused by Staph epi. Staph epi septicemia was considered to be present, when a) the newborns clinical condition deterioated, b) the blood culture was positive and c) the organism could be cultured from the catheter tip $(n=7)$, the tracheal aspirate (6) or a skin lesion (1).Additio nal signs of infection included an elevated body temperature to $38^{t} 0.35^{\circ}$ and a shift to nal signs of infection included an elevated body
the left in the white cell count with $11 \pm 8$ bands.

The mean birth weight of our pts is 1260 t 700 grs; the mean gestational age $31.2 \pm 4$ weeks. The mean 5 minute Apgar score was 7. ${ }^{ \pm 1}$.5. Eight pts were ventilated for $26.9 \pm 9$ days. Seven pts had a central venous catheter made of Silastic $R$ put in for a mean 17.4t12.3 days. In 4 pts the tracheal aspirate was contaminated by Staph epi $4.8 \mathrm{~d}^{2}$ days before the septicemia became clinically apparent and the organism could be cultured from the catheter tip.Six of eleven infections were associated with liver enlargment to 3 oms below the right costal margin; of those 3 had elevated liver enzymes and 3 had a mean bilinubin of $3.9 t_{1}$. Durring the same year we had 12 pts with similar gestational age, duration of ventilation and 5 minute Apgar score, who had a central venous catheter put in during antibiotic therapy mostly for maternal infection or prenature rupture of membranes. In those 12 we had 2 pts, in whom we cultured Staph epi from the catheter tip, but no pt developed clinical signs of infections or cholestasis. Conclusions:

Staph epi has been the most frequent organism causing septicenai in our NId.Pts with central venous lines or those, who were ventilated were at high risk of getting Staph epi septiceria, which was associated with liever enlargment. We recommend further prospec tive studies to clarify, wether cholestasis can be an early symptom of Staph epi septicenia.

\section{INFLUENCE OF FEEDING TUBES AND GASTROSTOMY ON THE COLONI-} H kraeft, R Roos, E Mrozik

Kinderklinik und Kinderchirurgische Klinik der Universität München, Munich, Germany

It is known that catheters in the upper digestive tract may alter the local flora. Therefore, we carried out bacterial counts on 165 gastric aspirates of neonates with gastrostomy and on 101 of neonates with feeding tubes. In the first group, organisms were found in $70 \%$, in the second group in $30 \%$ of all cases. Enterobacteriaceae, Enterococci, Pseudomonas aeruginosa and Candida albicans were isolated far cocci, Pseudomonas aeruginosa and Candida albicans were isolated
more frequently in the first group than in the second $(p<0,01)$. 6 infants with gastrostomy developed septicaemia caused by the same organisms that were also found in high numbers in the gastric aspirates. The influence of non-absorbable antibiotics in children with gastrostomy was studied prospectively in 72 gastric aspirates and 48 specimens of stool. There was no highly significant difference between infants with antibiotic treatment versus those without. It is concluded that gastrostomy influences the flora of the stomach and may conceivably lead to a septicaemia.
182 ENHANCED EFFICACY OF VIRA-A, ACYCLOVIR IN COMBINATION sacsohn M. (1) Rosenbluth M (1), S metana O (3), Rudenski B. (2) , Lipsical 1. (4) Doerner T. (4). Infectious Diseases (1) and (2) Microbiology Departments Shaare Zedek Medical Center Jerusalem; Department of Microbiology Sackler School of Medicine, Tel Aviv University (3) and Inter Yeda, Nes Ziona(4), Israel.

1. The efficacy of a single antiviral drug Vira-A, Acyclovir, Beta Interferon native and recombinant as compaired to a combination of two antiviral drugs acyclovir and eeta Interferon was evaluated in experimental herpes keratitis in rabbits. As a single antiviral drug experimental herpes keratitis in rabbits. As a single antiviral drug
acyclovir ointment promoted the most rapid epitelization time, but acyclovir ointment promoted the most rapid epitelization time, but
during the treatment period virus 'escapes' were detected. The best results were obtianed with a combination of Beta Interferon and acyclo vir demonstrating the potentiation of the antiviral activity of acyclovir by Beta Interferon. 2. In human herpes keratitis the combination of Beta Interferon and acyclovir resulted in a rapid epitelization time, short virus shedding time and no relapse during a subsequent 18 month observation period. 3. In human herpes labialis the combination of Beta Interferon and acyclovir resulted in a shortening of the disease to a $1 / 3$ of the usual period. Prophylactic Beta Interferon ointment when only numbling was felt prevented the appearance of vesicles on the lips. 4. In Herpes Zoster when started early in the disease the combination of acyclovir and Beta Interferon resulted in a shortening of disease and rapld dissappearance of pain.

\section{Incidence of P-fimbriated $\frac{E . c o l j}{\text { leaving neonatal wards in }}$ Sweden.}

Kjell Tullus, Lars Burman, Gunilla Källenius and Stefan B Svenson Pediatric Clinic, Danderyd Hospital and National Bacteriological Department, Sweden

We have previously shown that one P-fimbriated E.coli clone was spread in a neonatal ward. It could there colonize fecally many of the children and also members of the staff.

The children that were fecally colonized with the pyelonephritogenic, P-fimbriated E.coli clone ran a greater risk to develop pyelonephritis later on.

We have therefore studied all children leaving twenty-two different neonatal wards after the age of five days. From each child we characterized five fecal strains.

We found that a very high proportion (76\%) had klebsiella/enterobacter in their feces when they left the ward. In only $39 \%$ of all the children we found E.coli in the feces.

In most of the wards a few children leaving the ward had P-fimbriated E.colj in the stool. Two of the wards showed periods of time when many of the children leaving the ward were colonized with a P-fimbriated E.coli strain.

We now continue to study these two clinics both prospectively and retrospectively to see if this relatively high incidence of P-fimbriated E.coli among the children will lead to a higher incidence of pyelonephritis. 